\title{
Concrete Modification Using Graphene and Graphene Oxide
}

\author{
A. Mashhadani ${ }^{1}$, V. Pershin $*^{2}$ \\ ${ }^{1}$ Post-graduate student from the Republic of Iraq, Tambov State Technical University, 106 Sovetskaya Street, 392000, Tambov, Russia; \\ ${ }^{2}$ Department of Technology and Methods of Nanoproducts Manufacturing, Tambov State Technical University, \\ 106 Sovetskaya Street, 392000, Tambov, Russia \\ * Corresponding author. Tel.: +791065253 77. E-mail: pershin.home@mail.ru
}

\begin{abstract}
The paper considers methods for concrete modification using low-layer graphene and graphene oxide. It was found that graphene oxide and low-layer graphene obtained by liquid-phase shear exfoliation significantly increase the strength of concrete. In our opinion, low-layer graphene has better prospects since it is cheaper and its production technology is environmentally friendly. The viability and future of graphene largely depends on the availability of such a method that allows mass production of high-quality graphene at an affordable price. In this regard, liquid-phase separation of graphite with the formation of low-layer graphene, which can be used to modify concrete, turned out to be a competitive solution. The following main tasks were formulated in order to solve the organizational problems of low-layer graphene industrial production: reducing the concentration of low-layer graphene in concrete; increasing the concentration of low-layer graphene in suspension; developing an industrial technology for the production of concentrate with a high content of low-layer graphene; conducting full-scale experimental studies to determine the optimal concentration of low-layer graphene in concrete.
\end{abstract}

\section{Keywords}

Low-layer grapheme; graphene oxide; concrete; compressive strength; bending strength; water absorption; shear exfoliation.

(C) A. Mashhadani, V. Pershin, 2020

\section{Introduction}

Concrete is the most widely used composite material. A lot of research is aimed at improving its mechanical strength and durability, reducing water absorption and improving technological characteristics due to additives.

Environmental impact on cement materials, as a rule, leads to its degradation, in particular, increases brittleness and water absorption, which, of course, leads to the destruction of concrete structures.

As a rule, concrete has high compressive strength, but low bending strength, and a combination of these properties has a significant effect on durability. Improving the strength, especially the bending strength of concrete, has been the subject of many studies in recent decades. Conventional methods for increasing strength used the inclusion of reinforcements on a millimeter scale and/or on a micro scale using macro and micro fibers, respectively [1]. Since the cracking process arises and spreads at the nanoscale, the researchers used nanoscale reinforcements such as carbon nanotubes (CNTs), carbon nanofibres (CNFs) and nanoparticles [2-5], due to their high mechanical properties and the large surface area. Graphene, the recently appeared nanomaterial, exceeds these nanomaterials in terms of strength characteristics (the strength is $130 \mathrm{GPa}$ and Young's modulus is $1.0 \mathrm{TPa}$ ) measured using nanoindentation atomic force microscopy [6].

In combination with flying ash (FA - Fly Ash), which has environmental advantages and is a byproduct of coal-fired power plants, machinability, strength and resistance to chemical influences such as chlorides and sulfates are increased [7-12].

Due to the exceptional strength of graphene, it has been a subject of interest in reinforcing metal and polymer matrices [13-17]. Recent decades have begun to pay similar attention to strengthening ceramic matrices. However, as far as the authors know, only a few research works [18] were devoted to studying cement based on fly ash and graphene with respect to 
bending strength and resistance to chemical attack of mortars.

Much more attention was paid to graphene oxide as a concrete modifier. Apparently this is due to the fact that graphene oxide is already produced today on an industrial scale. Graphene oxide (GO) consists of a monolayer of $s p^{2}$ and $s p^{3}$ hybridized carbon atoms covalently linked to various functional groups, such as carboxyl, hydroxyl and epoxy [19, 20], which alter the van der Waals interactions between GO sheets, thereby improving its dispersion in water. Given that the tensile strength is $130 \mathrm{GPa}$ and the specific surface is $2630 \mathrm{~m}^{2} / \mathrm{g}$, as well as good dispersion in water, GO is a promising modifier for cement composites [21, 22].

\section{Using graphene oxide for concrete modification}

Sufficiently detailed studies of the GO effect on the concrete strength properties showed that $0.03 \mathrm{wt} . \%$ Gravel oxide increased the tensile strength by $49 \%$ [23]. Another study [24] stated that 15-33\% of compressive and tensile strengths were improved using 0.05 wt.\% GO in cement slurry by $41 \%$ and $59 \%$, respectively. These results were confirmed by other researchers $[25,26]$.

The dispersion quality plays an important role in the use of $\mathrm{GO}$ as a modifier for cement-based materials. The presence of more calcium ions in fresh cement paste causes decrease in the effectiveness of GO. Therefore, it is very important to study the dispersion mechanism of GO and to develop ways to improve the quality of dispersions.

In cement pastes, GO dispersion under the influence of calcium bivalent ions aggregates, which greatly reduces the modification efficiency. In order to remove GO aggregation in cement based materials, it is proposed to use silica smoke. It has been established that the dispersion quality improves not only for GO and for carbon nanotubes (CNTs) in cement paste by utilizing silica smoke $[27,28]$.

It was found that the fluidity of the cement slurry and the workability of concrete decreases when using GO [29]. This paper recommends the use of fly ash to increase the fluidity of the graphene oxide cement composite. It was experimentally established that the fluidity of a cement composite with graphene oxide increases with increasing amounts of fly ash. Improving the fluidity of graphene oxide-cement composite is due to the outstanding influence of the ball effect and the gradation of the fly ash grain size.

Based on a quantitative analysis of the rheological parameters, it was shown that fly ash can compensate for the decrease in GO fluidity. The effect of fly ash was studied with two doses of GO, 0.01 and 0.03 wt. \%
[30]. At 0.01 wt. $\%$ GO and 20 wt. \% fly ash, the yield strength of the paste decreased by $85.81 \%$ and the plastic viscosity decreased by $29.53 \%$ compared to the control sample (without fly ash or GO). At 0.03 wt. \% GO and 20 wt. \% fly ash, the yield strength of the paste was lower by $50.33 \%$, and the plastic viscosity decreased slightly by $5.58 \%$. Meanwhile, the results showed a good correlation between fluidity and plastic viscosity. In addition, GO can compensate for the delay in early strength gains in systems with fly ash and cement. When the dosage of fly ash is less than 15 wt. $\%$, the compressive and bending strength of the fly ash and GO cement composites is higher than that of the control sample after 3, 7 and 28 days. This indicates that the addition of fly ash is an economical and efficient way to obtain the desired properties of GO cement paste. The benefits of mixing fly ash and GO can help counteract the fluidity problems of GO cement paste for concrete workability.

In [31], the effect of additives of nanoplastic graphene oxide (GONS) on the properties of cement mortar and ultrahigh strength concrete (UHSC) was reported. The resulting GONS-cement composites were easy to prepare and showed excellent mechanical properties. However, their fluidity decreased with increasing GONS content. UHSC samples were prepared with different amounts of GONS (0-0.03 wt. \% cement). The results showed that when using 0.01 wt. $\%$ cement GONS, the compressive strength was $7.82 \%$ after 28 days of curing. Moreover, the addition of GONS improved bending and deformation strengths increasing bending strength more than compressive strength. FE-SEM observations showed that GONS were well dispersed in the matrix, and the bond between GONS and the surrounding cement matrix was strong. In addition, the FE-SEM study showed that GONS probably influenced the shape of cement hydration products. Nevertheless, the space for hydrate growth also had an important influence on the hydrate morphology. Therefore, the true mechanism of hydration of cement composites with GONS requires further study.

GO accelerates hydration [32] while temporarily delaying clinker hydration. This difference reflects the double behavior of GO in cement pastes. Slowdown occurs due to the interaction of GO with the surface of the moisturizing grains, and acceleration occurs due to the effect of sowing. Plaster causes this difference. It was shown that GO has a negligible effect on the strength of hardened pastes, and this simply refers to a change in the hydration degree, in contrast to the previously enhancing effect. In general, GO is not particularly active as a germinal surface, since it aggregates and behaves like inert fillers (e.g., quartz). 
A polycarboxylate-ether copolymer can make GO an active seed in cement pastes, since it prevents GO aggregation. However, it was discovered that this process occurs only in the alite paste, but not in the PC paste.

A new graphene oxide (GO) coated polyethylene (PE) fiber ( $\mathrm{GO} / \mathrm{PE}$ fiber) is being developed by simply mixing PE fibers in a GO aqueous solution at a certain temperature [33]. Experimental results show that, due to the different behavior of thermal expansion, shrinkage of GO at a higher temperature facilitates the formation of a three-dimensional coating around the surface of a polyethylene fiber. This will increase the surface wettability, roughness and chemical reactivity of the polyethylene fiber, greatly simplifying the physical and chemical interaction of GO/PE fibers with cement hydrates. Compared to control strainhardening cement composites (SHCC) with intact polyethylene fiber (2.0 vol. \%), the use of GO/PE fiber can improve the tensile strength and deformation ability of SHCC by 46.3 and $70.4 \%$ without compromising compressive strength, and the average crack opening width can be reduced from 138 to 58 microns. A noticeable improvement in the mechanical properties of SHCC is due to increased bonding of the fiber with the PE matrix due to the addition of GO, which is additionally confirmed by the results of a single fiber alignment test showing an increase in interfacial friction from 2.33 to $3.99 \mathrm{MPa}$. Finally, the micromechanical model is adopted to explain the mechanism for improving behavior during strain hardening. In conclusion, the research results provide an effective strategy for functionalizing the surface properties of $\mathrm{PE}$ fibers by applying GO coatings and for achieving stronger adhesion at the fiber / matrix interface, which leads to the development of a new high-strength SHCC with a tensile strength of up to $6 \%$.

Recent studies [34] have shown that graphene oxide (GO) can improve the mechanical properties of hardened Portland cement (PC) pastes. The mechanisms proposed so far to explain this enhancement usually suggest that GO is well dispersed in the pore solution of the PC paste serving as a reinforcing agent or nucleation site during hydration. This paper investigates (i) the effect of GO on the alite hydration, which is the main component of PC cement, using isothermal calorimetry and modeling of boundary growth, and (ii) factors controlling the colloidal stability of GO in the environment of alite paste. The results show that GO only slightly accelerates alite hydration and that GO is prone to aggregation in the alite paste. This instability is due to (i) a pH-dependent interaction between GO cations and calcium in the pore solution of the alite paste and (ii) a significant decrease in $\mathrm{GO}$ functional groups at high $\mathrm{pH}$.

An article [35] was the first to report the incorporation of in situ reduced graphene oxide (rGO) in geopolymers. Obtained rGO-geopolymer composites are easy to manufacture and have excellent mechanical properties. Geopolymers were prepared with graphene oxide (GO) concentrations of $0.00,0.10,0.35$ and 0.50 wt. \%. Functional groups, morphology, mechanisms for filling voids, and mechanical properties of composites were determined. Fourier transform infrared spectra (FTIR) showed that the alkaline solution reduces the hydroxyl/carbonyl groups of GO by deoxygenation and / or dehydration. At the same time, the spectral absorption associated with crosslinking such as silica increased in the spectra. Scanning electron microscope (SEM) micrographs showed that rGO changed the morphology of the geopolymers from a porous nature to a substantially pore-filled morphology with enhanced mechanical properties. Bending tests showed that $0.35 \mathrm{wt} . \% \mathrm{rGO}$ gave the highest bending strength, Young's modulus and bending viscosity, and they were increased by 134 , 376 and $56 \%$, respectively.

Hydration precursors, morphology, composition, and chemical bonds of cement pastes were studied using SEM / BSE, XRD, LA-ICP-MS and 29Si / 27Al MAS-NMR, respectively [36]. The experimental results showed that the 28-day compressive strength of cement pastes (weight $/ s=0.35$ ) increased by $29 \%$ with the addition of 0.02 wt. $\%$ GO. There were chemical reactions between mixed $\mathrm{GO}$ and cement hydration products. The increased strength is actually explained not only by the improved degree of cement hydration, but also by the recently discovered tobermorite-like hydrates and jennite-like hydrates due to the consumption of $\mathrm{Ca}$ ions by negatively charged GO. The polymerization of hydration products was also improved by the addition of GO.

The study [37] presented the development of a nano-cement composite with carbon nanomaterials based on GO synthesized from a high-purity epigenetic graphite deposit. Diamond drilling trial mineralization of graphite was improved by beneficiation and refining to recover a high purity graphite product $(99.9 \%$ graphite carbon " $\mathrm{Cg}$ "). An alternative and improved chemical oxidation process based on the modified Hammer method was adopted for the synthesis of GO from highly pure graphite. Microstructural analysis was performed to characterize GO. GO consists of functional groups $-\mathrm{OH},-\mathrm{C}=\mathrm{O},-\mathrm{COOH}$ and $\mathrm{COC}$ with a layer thickness of $1.2 \mathrm{~nm}, 2-3$ graphene layers, 
an interlayer distance of $0.90 \mathrm{~nm}$, and a Raman (ID / IG) ratio of 0.79 . The effect of $0.02,0.04$ and 0.06 wt. \% GO cement on the workability, hydration, microstructure, mechanical and transport properties of the composite was established. An increase in GO concentration in the composite decreased machinability due to the hydrophilicity of the 2D flat surface. The hydration rate was accelerated and the cumulative heat of hydration increased with increasing proportion of GO in the composite. Dosages of GO of about 0.02 and 0.04 wt. \% with respect to cement in composite materials led to a maximum increase in compressive and bending strength by 83 and $26 \%$, respectively, compared with the control mixture ( 0 wt. \% GO). Microstructural studies show that GO enhances the hydration of calcium hydroxide $(\mathbf{C H})$ and calcium silicate hydrate $(\mathrm{C}-\mathrm{S}-\mathrm{H})$ at the stages of nucleation and growth, fills the pores, connects microcracks and creates a relationship between the products of cement hydration. Together, these effects ultimately improve the mechanical properties of the composites. In addition, in this process, 0.02 and 0.04 wt. $\%$ GO of the cement composite increased the electrical resistivity by $11.5 \%$ and decreased the sorption capacity, respectively, by $29 \%$, which improved the overall characteristics of the composite.

The introduction of 0.05 wt. $\%$ GO can increase the compressive strength by $15-33 \%$ and the bending strength by 41-59\%, respectively [38]. Scanning electron microscopy of the GO-cement composite shows a high tortuosity of cracks indicating that a twodimensional GO sheet may form a barrier to crack propagation. Consequently, the GO-cement composite shows a wider stress-strain curve in the post-peak zone, which leads to less sudden failure. The addition of GO also increases the surface area of the GO-cement composite. This is due to the increased production of calcium silicate hydrate. The results from this study suggest that GO has the potential to be used as a nano-reinforcing additive in cement-based composite materials.

A new composite coating of graphene oxide (GO) and epoxy resin (EP) was proposed [39] to prevent the penetration of water and ions into the nanometer channel of a calcium silicate hydrate gel $(\mathrm{C}-\mathrm{S}-\mathrm{H})$. To assess the water resistance of the GO-EP composite coating, the local structure, dynamic properties, and the mechanism of water transfer by the moleculardynamic method were studied. Compared to a single graphene oxide coating, the migration of water and ions is significantly inhibited in the pores of $\mathrm{C}-\mathrm{S}-\mathrm{H}$ gel impregnated with $\mathrm{GO}$ and a hydrophobic epoxy composite. GO plays an important role in combining the $\mathrm{C}-\mathrm{S}-\mathrm{H}$ gel and the epoxy composite. The functional groups in the GO sheets bind to interfacial calcium atoms on the inner surface of the $\mathrm{C}-\mathrm{S}-\mathrm{H}$ gel and provide oxygen centers for accepting $\mathrm{H}$ bonds from the silicate tetrahedron. On the other hand, H-bonds transmitted by hydroxyl in GO sheets of epoxy resin help to strengthen the bond between GO sheets and epoxy molecules. During the process of capillary transport, water molecules are first dragged by hydroxyl groups on the GO sheets, accept or transfer their $\mathrm{H}$ bonds to the functional groups, and diffuse in the vacancy region between the epoxy molecules and the GO sheet. Hydrophobic epoxy molecules blocking the pores of the $\mathrm{C}-\mathrm{S}-\mathrm{H}$ gel inhibit the penetration of the solution. With a percentage of epoxy molecules from 21.8 to $87.2 \%$, the rotation speed of the molecule increases from 10.42 to 10.52 , and the penetration depth of water molecules slows down by $71.58 \%$. While the penetrating solution reduces the adhesion energy by $66.38 \%$ between the GO sheets and the epoxy group, the adhesion energy between $\mathrm{GO}$ and $\mathrm{C}-\mathrm{S}-\mathrm{H}$ remains constant. The incorporation of the $\mathrm{GO}$ sheet between $\mathrm{C}-\mathrm{S}-\mathrm{H}$ and epoxy may contribute to the stability of the composite coating. In addition, the mobility of $\mathrm{Na}$ and $\mathrm{Cl}$ ions decreases due to the presence of the GO-EP composite coating, which immobilizes the penetrated $\mathrm{Na}$ and $\mathrm{Cl}$ ions in the input region and separates the ions from the aqueous solution. We hope that the new hydrophilichydrophobic composite coating provides valuable information on the design of a waterproof material.

The effect of graphene oxide (GO) on the kinetics of hydration, pore structure, mechanical properties, and gel structure based on calcium silicate hydrate $(\mathrm{C}-\mathrm{S}-\mathrm{H})$ was systematically studied by combinatorial methods [40]. GO can accelerate cement hydration, improve pore structure, and increase the degree of polymerization of $\mathrm{C}-\mathrm{S}-\mathrm{H}$ gels due to the effects of GO nucleation. The specific surface area of the cement paste and the number of gel pores were reduced with the addition of GO. The threedimensional network structure of GO-modified $\mathrm{C}-\mathrm{S}-\mathrm{H}$ gels was originally proposed based on the results obtained, according to which $\mathrm{GO}$ can be introduced into the interlayer space of $\mathrm{C}-\mathrm{S}-\mathrm{H}$ gels by ion bonding with $\mathrm{Ca}_{2}{ }^{+}$and fill the pores of the gel. A small proportion of GO (0.022 wt. \%) increased compressive strength over 28 days by $16.31-25.60 \%$ at various ratios of water and cement, which indicates that GO is a potential nano-reinforcing material for cement composites. The results will provide a good understanding of the GO reinforcement mechanisms 
in cement composites and pave the way for the design of high-performance cement composites.

The efficient load transfer between the concrete base and fiber reinforced polymer (FRP) bonding agent is a key factor in any FRP reinforcement system [41]. Recently, the authors developed an innovative high-strength, self-sealing, non-polymer cement adhesive (IHSSC-CA), which has been used in a number of studies. Graphene oxide and cement materials are used to synthesize new adhesive. The successful implementation of IHSSC-CA significantly increases the utilization of the carbon FRP strip (CFRP) and the bearing capacity of the reinforcement system mounted on the NSM surface. A series of tests were performed to verify the interfacial zone in the area of bonding of NSM CFRP strips, including physical examination, analysis of pore structure and three-dimensional laser profilometry analysis. From a physical examination of NSR CFRP samples fabricated using IHSSC-CA, it was established that a smooth surface was found in the CFRP band to transfer the load without stress concentration in some local regions. The smooth surface of the adhesive layer is very important to prevent local brittle fracture in concrete. The analysis of pore structure also confirmed that IHSSC-CA has a better composite effect between NSM CFRP strips and the concrete substrate than other adhesives, as a result of which NSM CFRP samples made with IHSSC-CA can withstand a large load. Finally, the results of three-dimensional laser profilometry showed a greater degree of roughness and less deformation on the surface of the carbon fiber strip using IHSSC-CA, compared to other adhesives. Graphene oxide contains a number of reactive oxygen functional groups, which makes it a suitable candidate for the reaction in cement materials through physical functionalization. The aim of this work is to study the transport characteristics of cement composites reinforced with graphene oxide, which can be converted into concrete made from similar components. Transport characteristics determine the durability of concrete structures. Tests, such as water sorption, chloride penetration, and mercury porosimetry, were performed to observe the effect of the GO addition to the cement matrix and its transport properties. GO was dispersed in a cement mortar to obtain a GO-cement composite using additives of 0.01 , 0.03 and 0.06 wt. \% cement. Experimental results show that the introduction of a very low GO fraction $(0.01 \%)$ can effectively inhibit the penetration of chloride ions. In addition, the sorption capacity is significantly increased with the addition of GO with a moderate proportion of $0.03 \%$. It can be concluded that the addition of GO to the cement matrix can effectively improve the transport properties of the cement matrix, which subsequently improves its durability.

\section{Using low-layer graphene for concrete modification}

Graphene nanoplates have unique mechanical, thermal and electrical properties that make them ideal reinforcing materials. The attractive properties of graphene have led to intensive research on graphenepolymer nanocomposites. However, very little information was received on the use of graphene in the production of multifunctional cement-based nanocomposites. The article [42] highlights the main conclusions in the field of production and characteristics of graphene-cement nanocomposites (GCNC). At the atomic level, X-ray diffraction was used to predict chemical composition and crystallography. At the nanoscale level, atomic force microscopy (AFM) was used to study physical and chemical properties. Molecular dynamics analysis was carried out to assess the interfacial strength between calcium silicate hydrate $(\mathrm{C}-\mathrm{S}-\mathrm{H})$ and graphene nanoparticles functionalized with various chemical groups. At the micro level, scanning electron microscopy was used to obtain information on surface topography and the GCNC composition. At the mesoscale level, mechanical properties were measured using resonance ultrasound spectroscopy (RUS). This multiscale assessment showed a strong correlation between GCNC morphology and performance. The functionalization of graphene nanoplates tends to improve surface strength which leads to improving overall mechanical properties.

Experimental studies of transport properties under the influence of chlorides and water were carried out on concrete containing up to $2.5 \%$ graphene nanoplates (GNP) in increments of $0.5 \%$ [43]. The pore structure was obtained using mercury intrusion porosimetry, and a significant decrease in pore size was measured. Concrete with $1.5 \%$ GNP showed the greatest reduction in transport. The depth of water penetration, the diffusion coefficients of chlorides and migration were reduced by 80,80 and $37 \%$, respectively. The barrier effects of GNP have been characterized, and it has been found that more than $50 \%$ improvement in transport resistance may be due to tortuosity, while the rest is due to thinning pores. However, further improvement did not occur when the GNP content was above $1.5 \%$ due to the limited dispersion of nanoparticle clusters. 
GNP, a low-cost carbon nanoplast, was added to the solution at a content of $0,2.5,5.0$ and 7.5 wt. \% cement [43]. The water penetration depth, chloride diffusion coefficient and chloride migration were determined for GNP cement slurry and compared with pure cement slurry samples. The test results showed that the addition of $2.5 \%$ GNP can cause a significant decrease in the depth of water penetration, the diffusion coefficient of chloride and the migration coefficients of chloride by 64,70 and $31 \%$, respectively. A decrease in the penetration of water and ions can be partially associated with a decrease in the critical pore diameter by about $30 \%$. This refinement of the GNP microstructure is confirmed by the results of mercury intrusion porosimetry. Impervious GNP also contributes to reduced permeability due to increased tortuosity against penetration of water and aggressive ions.

The influence of the noncovalent surface modification method on the dispersion of graphene was shown in [44]. Nanoplastics (GNS) in water and chloride diffusion of the cement paste including these dispersions were investigated. The results showed that stable and homogeneous suspensions of graphene nanoplasts were obtained using polyoxyethylene (40) nonylphenyl ether (CO890) as a dispersant. In this study, the ratio of water to cement is 0.30 , the addition of GNS is from 0.02 to 0.15 wt. \% cement. It was found that introducing small amounts of GNS, only $0.02 \%$, can reduce chloride penetration and coefficient by as much as 37 and $42 \%$, respectively. This improvement can be explained by the increased degree of cement hydration, filling effect, the barrier effect and crack formation of the GNS effect in the cement matrix. This work provides a new way to further understand the improvement of GNS in cement composites.

To study the effect of GNS reinforcement on the mechanical properties, pore structure and electrical characteristics of hardened cement composites, GNS was added in various percentages of $0,0.01,0.02$, $0.03,0.04$, and 0.05 wt. $\%$ in relation to cement [45]. The compressive strength was determined on the 28th day of curing. Thermogravimetric analysis (TGA) was used to detect phase decomposition, the pore structure was studied using nitrogen adsorption at $77.35 \mathrm{~K}$, the microstructure was analyzed by scanning electron microscopy (SEM), and finally, the electrical conductivity of graphene-cement composites was also studied. The results showed that a significant increase in compressive strength of approximately $33 \%$ was achieved by incorporating GNS into the cement matrix by approximately $0.04 \%$. The integration of graphene in cement significantly reduced the pore size of the pastes and led to a significant improvement in the microstructure with subsequent improvement in the electrical conductivity of these composites.

Cylindrical samples with a diameter of 50 and $100 \mathrm{~mm}$ were made from solutions with a water/ cement ratio of 0.4 and the addition of multilayer graphene (MLG) from 0.015 to $0.033 \%$, by weight of cement and sand [46].

The thickness of graphene particles was from 2 to $20 \mathrm{~nm}$. Tensile and compression tests were performed at 3, 7, and 28 days of age. In addition, SEM micrographs and metallographic images were collected and analyzed to better characterize the morphology of the sample, as well as to explain the effect of MLG on the cement strength. The optimum tensile strength was achieved with samples developed with a MLG dose of $0.033 \%$, for which a corresponding increase was 100.0, 144.4 and $131.6 \%$ after 3,7 and 28 days, respectively, compared with samples without MLG. On the other hand, optimal compressive strength was obtained at MLG concentrations of $0.021,63.6,94.1$ and $95.7 \%$ after 3,7 and 28 days, respectively, compared with samples without MLG addition. It is believed that MLG accelerates cement hydration reactions, reduces pore volume and hardens cement properties.

Cement-based composites made from graphene nano-platelets (GN) and hollow glass microspheres (HGM) and its properties of electromagnetic wave absorption were investigated [47].

The results show that the absorption properties were improved after a combination of GN and HGM. With an increase in the coefficient of filling with glass microspheres, the magnitude of the absorption peak and the bandwidth below $5 \mathrm{~dB}$ first increase and then decrease. In addition, several sharp peaks were obtained and the values tend to appear at high frequency. With a further increase in $\mathrm{GN}$, the value at the absorption peak decreases and the curves become relatively flatter. When $\mathrm{GN}$ is $0.2 \%$, HGM is $40 \%$ (vol.) and thickness is $20 \mathrm{~mm}$, the materials have excellent absorbing properties, with an average reflectivity loss of $8.2 \mathrm{~dB}$ in the range $2-18 \mathrm{GHz}$, and the bandwidth was $4.4 \mathrm{GHz}$ below $5 \mathrm{~dB}$. The thickness of the sample has a significant effect on absorbency. The optimum thickness is $20-30 \mathrm{~mm}$ with $40 \% \mathrm{HGM}$ and $0.2 \% \mathrm{GN}$.

Electrochemically exfoliated graphene (EEG) is a hybrid functional material that has a significantly improved microstructure and mechanical properties, as well as constant machinability [48]. In terms of characteristics, it surpasses previously reported cement 
composites containing graphene derivatives. The manufacture of the composite is based on a simple and effective method, which allows to uniformly disperse the EEG in the cement matrix in the absence of surface-active substances. It was found that, unlike graphene oxide, EEG does not agglomerate in a cement-alkaline environment, which does not affect the fluidity of cement composites. The addition of 0.05 wt. $\%$ Graphene to conventional Portland cement results in an increase of tensile strength, compressive strength and Young's modulus by 79,8 and $9 \%$, respectively. It is noteworthy that the addition of EEG promotes the hydration reaction of both alite and belite, which leads to the formation of a large fraction of the $3 \mathrm{CaO} \cdot 2 \mathrm{SiO}_{2} \cdot 3 \mathrm{H}_{2} \mathrm{O}(\mathrm{C}-\mathrm{S}-\mathrm{H})$ phase. These results represent an important step forward in the practical application of nanomaterials in civil engineering.

Graphene nanoplates (GNPs) and graphene oxide nanoplates (GONPs) are promising nanoscale additives for improving the mechanical properties and strength of cement materials [49]. To further determine the collective influence of GNPs and GONPs, in particular their content, on concrete performance, a comprehensive study is conducted in this paper. Solution samples characterized by different water-cement ratios and containing different amounts of GNPs and GONPs undergo experimental tests to systematically study their strength, electrical resistance and piezoresistive reactions. The results show that in order to take advantage of GNPs and GONPs, the ratio of water cement and the appropriate workability of concrete are important. With an appropriate water-cement ratio, a small percentage of GNPs and GONPs can significantly improve concrete strength, while the content of GNPs needs to be significantly increased to achieve low electrical resistance and an accurate piezoresistive response. To understand the mechanisms of electrical conductivity and piezoresistance at the micro level, a microcharacterization is carried out using scanning electric microscopy (SEM), equipped with the method of energy dispersive spectrometer (EMF), on order to study the microstructure of a solution containing GNPs. In addition, a quick chloride corrosion test on selected mortar samples shows that chloride ion migration slows down with the addition of GNPs to the cement paste.

The effect of graphene and GO on hydration, microstructure, and mechanical properties of cement paste was first studied in [50]. The introduction of 0.16 wt. $\%$ GO into the cement matrix can increase the bending strength of the material by $11.62 \%$ due to the higher degree of hydration, the effect of nanofiller and the effect of the formation of cracks and bridges. On the other hand, graphene reduces the development of hydration and the mechanical properties of cement paste due to its poor dispersibility in an alkaline environment. In addition, the various interaction mechanisms between Graphene/GO and cement hydrates have been deeply studied using molecular dynamics (MD) of reactive force field, which showed that functional hydroxyl groups in GO provide nonbridged oxygen (NBO) sites that accept hydrogen interlayer bonds water molecules in calcium silicate hydrate $(\mathrm{CeSeH})$. In the presence of interface counterions, protons are transferred from $-\mathrm{OH}$ to $\mathrm{GO}$ to $\mathrm{NBO}$ sites in $\mathrm{CeSeH}$, which additionally contributes to the polarity of the GO surface and strengthens the bond with neighboring substances. In addition to H-bond bonds, $\mathrm{Ca}_{2}$ and $\mathrm{Al}_{3}$ ions near the $\mathrm{CeSeH}$ surface play an intermediary role in the bridge of oxygen atoms in silicate chains and hydroxyl groups in GO, which increases the length of the silicate chain and treats the defective GO structure. Dynamically aluminate-silicate chains, calcium ions and functional hydroxyl groups form "cells" and strictly prevent the free diffusion of water molecules at the interface stabilizing the bonds between $\mathrm{CeSeH}$ and GO structures. Finally, uniaxial tensile modeling showed that while high adhesion and ductility in the GO-modified cement composite are mainly provided by strong structural $\mathrm{H}$-bonds and the calcium aluminate framework, the weakest mechanical behavior of the grapheme / $\mathrm{CeSeH}$ composite is explained by poor bonding and instability atoms in the interface. The reactive force field combines the chemical and mechanical reactions that occurred during the water dissociation, the exchange of protons between the $\mathrm{CeSeH}$ and GO structures, and the silicate-aluminatecarbon network dimerized to resist tensile loading.

The study [51] compares the hydration, mechanical and transport properties of cement-based composites containing graphene oxide (GO), reduced graphene oxide (rGO), and pure graphene (G). The only variable design of the mixture, different from the type of graphene material under consideration (GO, rGO and $\mathrm{G})$, is the concentration of graphene materials, which is in the range from 0.01 to $0.16 \mathrm{wt}$. \% cement. The results of this study indicate that the physical and chemical properties of $\mathrm{GO}, \mathrm{rGO}$, and $\mathrm{G}$ affect the early age properties of cement based composites. A high amount of GO oxygen-containing functional groups significantly enhances cement hydration and forms a densified composite bond, which improves the compressive and bending strength for 14 days by 44 and $83 \%$, respectively, compared with the control 
mixture. The reduced number of functional groups and moderately high physical strength of rGO gradually increase cement hydration and strengthen the composite matrix, which improves the 14-day compressive and bending strength by 42 and $52 \%$, respectively, compared with the control mixture. High physical strength $G$ strengthens the composite matrix, which improves the 14-day compressive and bending strengths by 34 and $56 \%$, respectively, compared to the control mixture. The microscopic image and electrical resistivity characteristics also confirm the effect of graphene materials on the composite structure.

The article [52] is also of greatest interest where the results of concrete modification with two types of graphene functionalized with surface-active graphene (FG) and commercially available graphene nanoplates (IG) are presented. To introduce the multifunctionality of concrete using graphene (FG), graphene suspensions in water were first prepared by liquid-phase exfoliation of graphite powder using a surfactant choleate [53].

Suspensions with industrial graphene (IG) were also prepared using a high shear stator-rotor mixer.

The inclusion of ultrathin graphite granules (UTGr) in concrete obtained by the same method as FG was also examined to determine whether graphene or graphite is the most effective nanoscale for concrete. It was found that graphene FG consisted of flakes with a number of layers ranging from four to seven, with most flakes having a thickness of six graphene layers. Graphene IG consisted of flakes with a quantity layers range from 10 to 14 with most flakes containing 10-11 graphene layers. UTGr consisted of flakes with a majority of 20-21 layers. Graphene was added to concrete by mixing dispersions of water-based graphene with ordinary Portland cement (OPC), fine dry sand and a $10 \mathrm{~mm}$ large aggregate. Fresh concrete mix was poured into standard steel molds measuring $10 \times 10 \times 10 \mathrm{~cm}$, then removed after 24 hours and held in the water tank so that the concrete finally hardened. Reinforced graphene samples were compared with standard concrete. For this purpose, a group of control samples was created according to the same procedure, but with the replacement of graphene aqueous solution with ordinary tap water. For bending tests, beams of $10 \times 10 \times 40 \mathrm{~cm}$ were prepared.

Fig. 1 shows the characteristic dependence of the compressive strength on the graphene concentration in suspension.

The graph shows that there is a pronounced extreme maximum at a concentration of $0.8 \mathrm{~g} / 1$. In [52], there is an even greater extremum equal to $47 \mathrm{MPa}$ at a concentration of $0.7 \mathrm{~g} / 1$, however, we believe that this is a random variable, the occurrence of which can be explained by a small number of experiments (only 3 samples were tested in compression work).

Fig. 2 shows the dependence of bending strength on the graphene concentration in suspension.

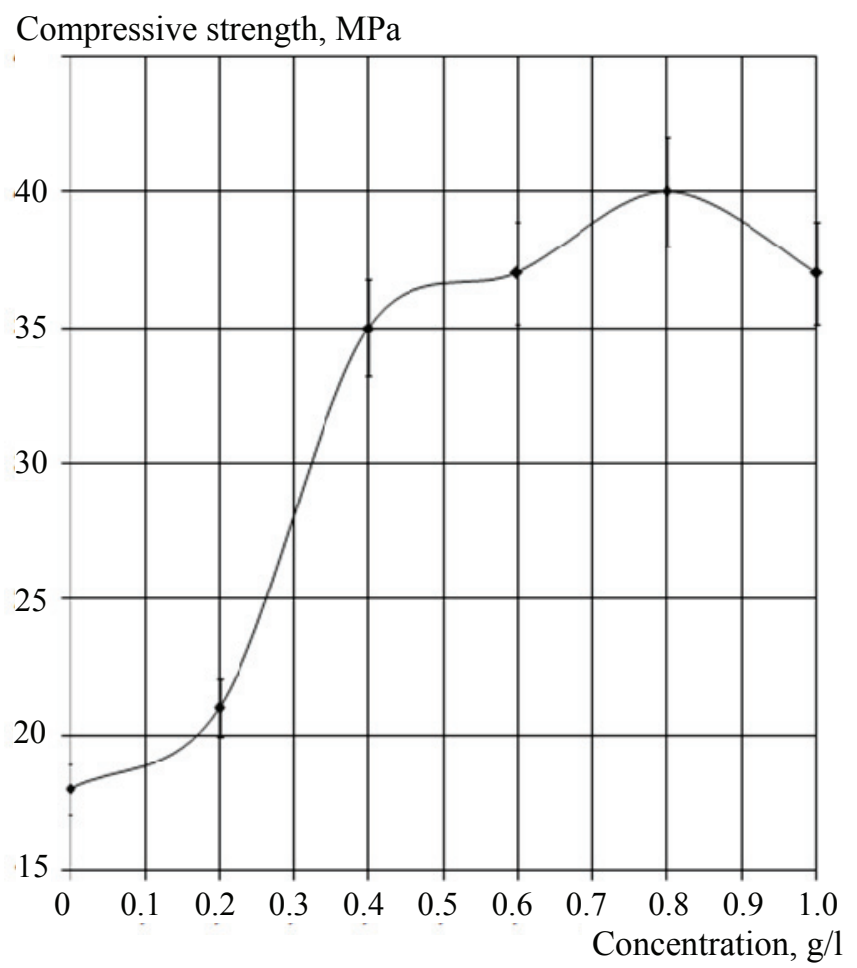

Fig. 1. Dependence of the compressive strength on the graphene concentration in suspension

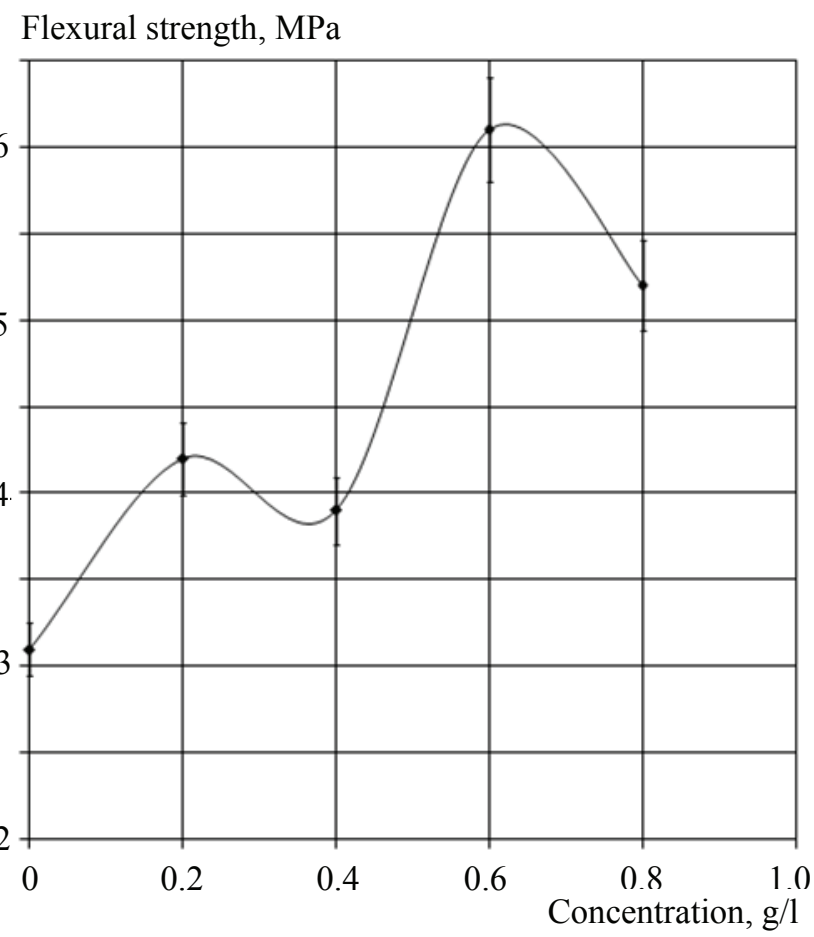

Fig. 2. Dependence of bending strength on the graphene concentration in suspension 
Compressive strength, $\mathrm{MPa}$

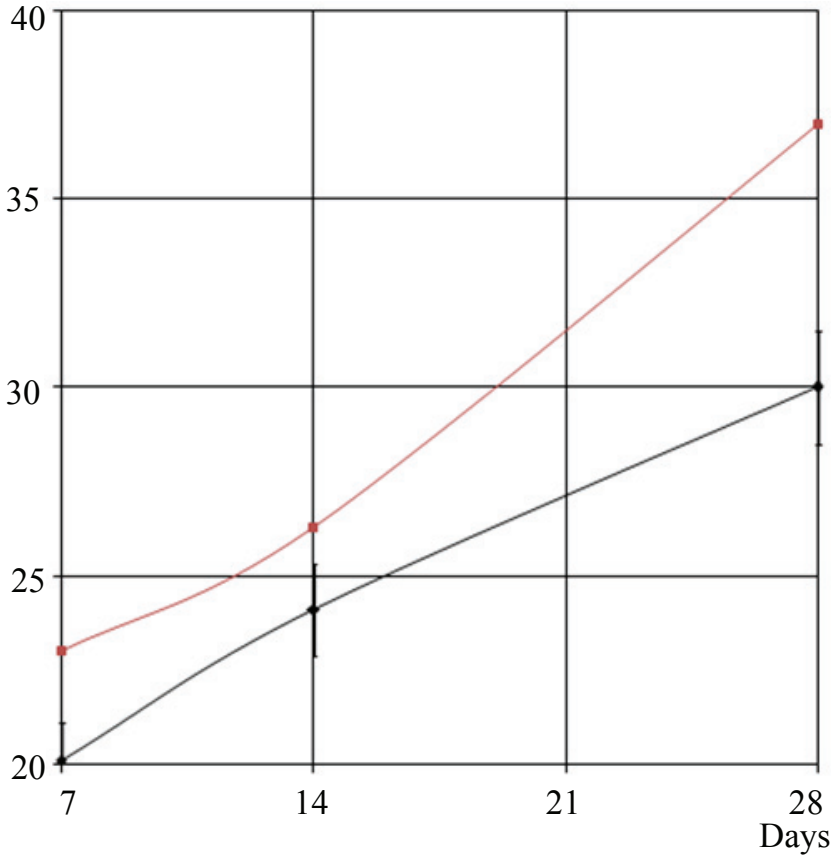

Fig. 3. Dependence of the compressive strength on the curing time of concrete

As in the case of compression, this dependence has an extremum maximum at the graphene concentration of $0.6 \mathrm{~g} / \mathrm{l}$ in suspension. It is clear that the decrease in strength at a concentration of $0.4 \mathrm{~g} / 1$ is random.

Fig. 3 shows the characteristic dependence of the compressive strength on the curing time of concrete. The lower curve corresponds to standard concrete, and the upper one to concrete which was prepared using a suspension containing $0.7 \mathrm{~g} / 1$ graphene.

The dependencies show that as a result of the concrete modification using low-layer graphene, the compressive strength is increased by $27 \%$.

Particular attention should be paid to the inconsistency of Fig. 1 and 3. In Fig. 1, at a zero concentration of graphene, the compressive strength is $18 \mathrm{MPa}$, and in Fig. $3-30 \mathrm{MPa}$. The article did not explain this discrepancy. In addition, the concentration of low-layer graphene of $0.7 \mathrm{~g} / \mathrm{l}$ is not economically viable. Indeed, for the preparation of one cubic meter of concrete, as a rule, about 170 liters of water are required. Thus, the preparation of one cubic meter of concrete requires $120 \mathrm{~g}$ of graphene. World cement production in 2019 was about 4,000 million tons. It is not difficult to imagine how much concrete is produced annually and how much low-graphene is required. In addition, the concentration in the suspension is low (1 g/l [53]). Therefore, it is extremely unprofitable to transport this suspension from specialized plants. Thus, it is necessary to develop an industrial technology for the production of concentrates with a high content of low-layer graphene.

\section{Conclusion}

The analysis shows that graphene oxide and lowlayer graphene obtained by liquid-phase shear exfoliation significantly increase the strength of concrete. In our opinion, low-layer graphene has better prospects, since it is cheaper and its production technology is environmentally friendly. The viability and future of graphene largely depends on the availability of such a method that allows mass production of high-quality graphene at an affordable price. In this regard, liquid-phase separation of graphite with the formation of low-layer graphene, which can be used to modify concrete, turned out to be a competitive solution. At the same time, there is a number of problems that must be solved:

- reducing the concentration of low graphene in concrete;

- increasing the concentration of low-layer graphene in suspension;

- developing an industrial technology for the production of concentrate with a high content of lowlayer graphene;

- conducting full-scale experimental studies to determine the optimal concentration of low-layer graphene in concrete.

\section{References}

1. Akkaya Y., Shah S.P., Ghandehari M. Influence of Fiber Dispersion on the Performance of Microfiber Reinforced Cement Composites. Am. Concr. Inst., 2016, 216, 1-18.

2. Makar J.M., Beaudoin J.J. Carbon Nanotubes and their Applications in the Construction Industry. Proceedings of the 1st International Symposium on Nanotechnology in Construction, Paisley, Scotland, 2003, 331-341.

3. Li H., Xiao H.G., Yuan J., Ou J. Microstructure of Cement Mortar with Nanoparticles. Compos, Part B, 2004, 35 (2), 185-189.

4. Li G. Properties of High-volume Fly Ash Concrete Incorporating Nano- $\mathrm{SiO}_{2}$. Cem. Concr. Res., 2004, 34 (6), 1043-1049.

5. Han B., Guan X., Ou J. Specific Resistance and Pressure Sensitivity of Cement Paste Admixing with Nano$\mathrm{TiO}_{2}$ and Carbon Fiber. Ceram. Soc., 2004, 32 (7), 884-887.

6. Lee C., Wei X., Kysar J.W., Hone J. Measurement of the Elastic Properties and Intrinsic Strength of Monolayer Graphene. Science, 2008, 321(5887), 385-388.

7. Langan B.W., Weng K., Ward M.A. Effect of Silica Fume and Fly Ash on Heat of Hydration of Portland Cement. Cem. Concr. Res., 2002, 32, 1045-1051.

8. Saraswathy V., Song H.W. Evaluation of Corrosion Resistance of Portland Pozzolana Cement and Fly Ash Blended Cements in Pre-cracked Reinforced Concrete Slabs under Accelerated Testing Conditions. Mater. Chem. Phys., 2007, 104, 356-361. 
9. Yen T., Hsu T.H., Liu Y.W., Chen S.H. Influence of Class F Fly Ash on the Abrasionerosion Resistance of Highstrength Concrete. Constr. Build. Mater., 2007, 21, 458-463.

10. Arora S., Singh S.P. Fatigue Strength and Failure Probability of Concrete Made with RCA. Mag. Concrete Res., 2017, 69 (2), 55-67.

11. Miranda J.M., Jimenez A.F., Gonzalez J.A., Palomo A. Corrosion Resistance in Activated Fly Ash Mortars. Cem. Concr. Res., 2005, 35, 1210-1217.

12. Yazici H. The Effect of Silica Fume and Highvolume Class C Fly Ash on Mechanical Properties, Chloride Penetration and Freeze-thaw Resistance of Self-compacting Concrete. Constr. Build. Mater., 2008, 22, pp. 456-462.

13. Sadowski P., Gajewska K.K., Stupkiewicz S. Classical Estimates of the Effective Thermoelastic Properties of Copper-Graphene Composites. Compos. Part B: Eng., 2015, 80, 278-290.

14. Liu X., Wei D., Zhuang L., Cai C., Zhao Y. Fabrication of High-strength Graphene Nanosheets/Cu Composites by Accumulative Roll Bonding. Mater. Sci. Eng.: A, 2015, 642, 1-6.

15. Li C., Hou T., She X., Wei X., She F., Gao W., Kong L. Decomposition Properties of PVA/Graphene Composites During Melting-Crystallization. Polym. Degrad. Stabil., 2015, 119, 178-189.

16. Liang J.Z., Du Q., Wei L.Y., Tsui C.P., Tang C.Y., Law W.C., Zhang S.D. Melt Extrudate Swell Behavior of Graphene Nano-Platelets Filled-Polypropylene Composites. Polym. Test, 2015, 45, 179-184.

17. Nguyen V.H., Shim J.J. Ionic Liquid-assisted Synthesis and Electrochemical Properties of Ultrathin $\mathrm{Co}_{3} \mathrm{O}_{4}$ Nanotube-intercalated Graphene Composites, Mater. Lett., 2015, 157, 290-294.

18. Sharma Snigdha, Arora Sumit. Economical Graphene Reinforced Fly Ash Cement Composite Made with Recycled Aggregates for Improved Sulphate Resistance and Mechanical Performance. Construction and Building Materials, 2018, 162, 608-612. Available at: https://doi.org/10.1016/j.conbuildmat

19. Wu L., Liu L., Gao B., Muñoz-Carpena R., Zhang M., Chen H., Zhou Z., Wang H. Aggregation Kinetics of Graphene Oxides in Aqueous Solutions: Experiments. Mech. Model. Langmuir, 2013, 29 (49), pp. 15174-15181.

20. Zhu Y., Murali S., Cai W., Li X., Ji W.S., Potts J.R., Ruoff R.S. Graphene and Graphene Oxide: Synthesis, Properties, and Applications. Adv. Mater., 2010, 22 (46), 3906-3924.

21. Zhou J., Wen H., Zhang Z., Luo D., Gao D., Yang J., Liang D., Li Y. The Dispersion and Aggregation of Graphene Oxide in Aqueous Media. Nanoscale, 2016, 8, pp. 14587-14592.

22. Li X., Liu Y.M., Li W.G., Li C.Y., Sanjayan J.G., Duan W.H., Li Z. Effects of Graphene Oxide Agglomerates on Workability, Hydration, Microstructure and Compressive Strength of Cement Paste. Constr. Build. Mater., 2017, $145,402-410$.
23. Gong K. et al. Reinforcing Effects of Graphene Oxide on Portland Cement Paste. Civ. Eng., 2014.

24. Pan Z. et al. Mechanical Properties and Microstructure of a Graphene Oxidecement Composite. Cem. Concr. Compos., 2015, 58, 140-147.

25. Lv S. et al. Effect of GO Nanosheets on Shapes of Cement Hydration Crystals and their Formation Process. Constr. Build. Mater., 2014, 64, 231-239.

26. Lv S. et al. Effect of Graphene Oxide Nanosheets on Microstructure and Mechanical Properties of Cement Composites. Constr. Build. Mater., 2013, 49, 121-127.

27. Sanchez F., Ince C. Microstructure and Microscopic Properties of Hybrid Carbon Nanofiber/Silica Fume Cement Composites. Compos. Sci. Technol., 2009, 69 (7), 1310-1318.

28. Kim H., Nam I., Lee H. Enhanced Effect of Carbon Nanotube on Mechanical and Electrical Properties of Cement Composites by Incorporation of Silica Fume. Compos. Struct., 2014, 107, 60-69.

29. Qin Wang et al. Effect of Fly Ash on Rheological Properties of Graphene Oxide Cement Paste. Constr. Build. Mater., 2017, 138, 35-44.

30. Qin Wang, Xinyou Cui, Jian Wang, Shiyu Li, Chunxiang Lv, Yichen Dong Effect of Fly Ash on Rheological Properties of Graphene Oxide Cement Paste. Construction and Building Materials, 2017, 138, 35-44. Available at: http://dx.doi.org/10.1016/j.conbuildmat. 2017.01.126

31. Liulei Lu, Dong Ouyang. Properties of Cement Mortar and Ultra-High Strength Concrete Incorporating Graphene Oxide Nanosheets. Nanomaterials, 2017, 7, 187, doi:10.3390/nano7070187.

32. Sam Ghazizadeha, Philippe Duffoura, Neal T. Skipperb, Yun Baia. Understanding the Behaviour of Graphene Oxide in Portland Cement Paste. Cement and Concrete Research, 2018, 111, 169-182. Available at: https://doi.org/10.1016/j.cemconres.2018.05.016

33. Zeyu Lu, Jie Yao, Christopher K., Leung Y. Using Graphene Oxide to Strengthen the Bond Between PE Fiber and Matrix to Improve the Strain Hardening Behavior of SHCC. Cement and Concrete Research, 2019, 126, 105899. Available at: https://doi.org/10.1016/j.cemconres.2019.105899

34. Ghazizadeha S., Duffoura P., Skipperb N.T., Billingb M., Baia Y. An Investigation into the Colloidal Stability of Graphene Oxide Nano-layers in Alite Paste. Cement and Concrete Research, 2017, 99, pp. 116-128. Available at: https://doi.org/10.1016/j.cemconres.2017.05.011

35. Mohamed Saafia, Leung Tangb, Jason Funga, Mahbubur Rahmana, John Liggatc. Enhanced Properties of Graphene/Fly Ash Geopolymeric Composite Cement. Cement and Concrete Research, 2015, 67, 292-299. Available at: https://doi.org/10.1016/j.cemconres.2014.08.011

36. Gang $\mathrm{Xu}$, Sen $\mathrm{Du}$, Jialuo $\mathrm{He}$, Xianming Shi. The Role of Admixed Graphene Oxide in a Cement Hydration System. Carbon, 2019, 148, 141-150, doi: 10.1016/j.carbon.2019.03.072. 
37. Tanvir S. Qureshi, Daman K. Panesar, Boopathi Sidhureddy, Aicheng Chen, Peter C. Wood. Nano-cement Composite with Graphene Oxide Produced from Epigenetic Graphite Deposit. Composites Part B, 2019, 159, 248-258.

38. Zhu Pan, Li He, Ling Qiu, Asghar Habibnejad Korayem, Gang Li, Jun Wu Zhu, Frank Collins, Dan Li, Wen Hui Duan, Ming Chien Wang. Mechanical Properties and Microstructure of a Graphene Oxide-cement Composite. Cement \& Concrete Composites, 2015, 58, 140-147.

39. Jiao Yu, Qi Zheng, Dongshuai Hou, Jinrui Zhang, Shaochun Li, Zuquan Jin, Pan Wang, Bing Yin, Xinpeng Wang. Insights on the Capillary Transport Mechanism in the Sustainable Cement Hydrate Impregnated with Graphene Oxide and Epoxy Composite. Composites Part B, 2019, 173, 106907.

40. Li Zhao, Xinli Guo, Yuanyuan Liu, Yuhong Zhao, Zhongtao Chen, Yunsheng Zhang, Liping Guo, Xin Shu, Jiaping Liu. Hydration Kinetics, Pore Structure, 3D Network Calcium Silicate Hydrate, and Mechanical Behavior of Graphene Oxide Reinforced Cement Composites. Construction and Building Materials, 2018, 190, 150-163.

41. Alyaa Mohammed, Nihad Tareq Khshain Al-Saadi, Riadh Al-Mahaidi. Assessment of Bond Strength of NSM CFRP Strips Embedded in Concrete Using Cementitious Adhesive Made with Graphene Oxide. Construction and Building Materials, 2017, 154, 504-513.

42. Materials Genome for Graphene-Cement Nanocomposites. Journal of Nanomechanics and Micromechanics, 2013, 3 (3), 67-77, doi: 10.1061/(ASCE) NM.2153-5477.0000055

43. Hongjian Du, Hongchen Jacey, Gao Sze, Dai Pang. Improvement in Concrete Resistance Against Water and Chloride Ingress by Adding Graphene Nanoplatelet. Cement and Concrete Research, 2016, 83, 114-123, doi: 10.1016/j.cemconres.2016.02.005

44. Baomin Wang, Ruying Zhao. Effect of Graphene Nano-sheets on the Chloride Penetration and Microstructure of the Cement Based Composite. Construction and Building Materials, 2017, doi: 10.1016/j.conbuildmat.2017.12.094.

45. Enhancement of Physico-Mechanical Characteristics, August 2017, doi: 10.23956/ijermt.v6i8.121.

46. Rodrigo Alves e Silva, Paulo de Castro Guettia, Mário Sérgio da Luz, Francisco Rouxinol, Rogério Valentim
Gelamo. Enhanced Properties of Cement Mortars with Multilayer Graphene Nanoparticles. Construction and Building Materials, 2017, 149, 378-385, doi: 10.1016/j.conbuildmat.2017.05.146.

47. Xingjun Lv, Yuping Duan, Guoqing Chen. Electromagnetic Wave Absorption Properties of Cementbased Composites Filled with Graphene Nano-platelets and Hollow Glass Microspheres. Construction and Building Materials, 2018, 162, 280-285, doi: 10.1016/ j.conbuildmat.2017.12.047.

48. High-Performance Graphene-Based Cementitious Composites, 2019, doi: 10.1002/advs.201801195.

49. Qiong Liu, Qingfeng Xu, Qiang Yu, Rundong Gao, Teng Tong. Experimental Investigation on Mechanical and Piezoresistive Properties of Cementitious Materials Containing Graphene and Graphene Oxide Nanoplatelets. Construction and Building Materials, 2016, 127, 565-576.

50. Dongshuai Hou, Zeyu Lu, Xiangyu Li, Hongyan Ma, Zongjin Li. Reactive Molecular Dynamics and Experimental Study of Graphene Cement Composites: Structure, Dynamics and Reinforcement Mechanisms. Carbon, 2017, 115, 188-208.

51. Tanvir Qureshi, Daman K. Panesar. A Comparison of Graphene Oxide, Reduced Graphene Oxide and Pure Graphene: Early Age Properties of Cement Composites. 2nd RILEM Spring Convention \& International Conference on Sustainable Materials, Systems and Structures (SMSS2019), At Rovinj, Croatia, 2019. Available at: https://www.researchgate.net/publication/331483393_A_com parison_of_graphene_oxide_reduced_graphene_oxide_and_pu re_graphene_early_age_properties_of_cement_composites.

52. Dimov D., Amit I., Gorrie O., Barnes M.D., Townsend N.J., Neves A.I., Withers F., Russo S., Craciun M.F. Ultrahigh Performance Nanoengineered Graphene-Concrete Composites for Multifunctional Applications. Adv. Funct. Mater., 2018, 28, 1705183.

53. Paton K.R., Varrla E., Backes C., Smith R.J., Khan U., O’Neill A., Boland C., Lotya M., Istrate O.M., King P., Higgins T., Barwich S., May P., Puczkarski P., Ahmed I., Moebius M., Pettersson H., Long E., Coelho J., O’Brien S.E., McGuire E.K., Sanchez B.M., Duesberg G.S., McEvoy N., Pennycook T.J., Downing C., Crossley A., Nicolosi V., Coleman J.N. Nat. mater., 2014, 13(6), 3944. 